\title{
Moderate Rectal Dysplasia
}

National Cancer Institute

\section{Source}

National Cancer Institute. Moderate Rectal Dysplasia. NCI Thesaurus. Code C4852.

A morphologic finding indicating the presence of moderate dysplastic cellular changes and moderate architectural changes in the glandular epithelium of the rectal mucosa. There is no evidence of invasion. 